rev.relac.int.estrateg.segur.8(1):309-326,2013

\title{
LA COOPERACIÓN INTERNACIONAL PARA EL CONTROL DEL TRÁFICO DE ARMAS, MUNICIONES Y EXPLOSIVOS: UN MECANISMO PARA LA GENERACIÓN DE SEGURIDAD ONTOLÓGICA EN COLOMBIA*
}

\author{
Carlos Alberto Ardila Castro** \\ Carlos Antonio Pinedo Herrera***
}

\section{RESUMEN}

Aunque los resultados de la participación de Colombia en los mecanismos de cooperación internacional dirigidos al control del tráfico de armas, municiones y explosivos, generalmente son analizados en relación con los efectos físicos en términos de la reducción de homicidios y el incautamiento de armas ilegales, es necesario que se identifiquen otras esferas igualmente importantes para la construcción de

* Este artículo es producto de la investigación Identificación y evaluación de impacto de las medidas aplicadas por parte de Colombia en el marco de los mecanismos de cooperación contra el tráfico de armas, municiones y explosivos, elaborado por Carlos Alberto Ardila Castro, para optar al titulo de magister en Relaciones y Negocios Internacionales. Universidad Militar Nueva Granada.

** Profesional en ciencias Militares, Gerente en seguridad y Análisis Sociopolítico, Magister en Relaciones y Negocios Internacionales, Especialista en Estudios Políticos, Consultor del Centro de las Naciones Unidas contra Tráfico de Armas Municiones y Explosivos, el desarrollo y la paz para Latinoamérica y el Caribe. Carlosardilacastro@gmail.com

*** Profesional en Ciencias Navales, Especialista en Comando y Estado Mayor, Especialista en Seguridad y Defensa Nacional, Especialista en Administración de la Seguridad Integral, Magister en Relaciones y Negocios Internacionales, fue Consultor de la Embajada del Reino de Suecia en Colombia para temas de desarme, desmovilización y reinserción, actualmente Docente de Planta y director del Programa de Administración de la Seguridad y Salud Ocupacional en la Facultad de Relaciones Internacionales Estrategia y Seguridad de la universidad Militar Nueva Granada.carlos.pinedo@unimilitar.edu.co 
seguridad en el país, dentro de los que se incluye el aspecto identitario y el reconocimiento de la posición de Colombia en el escenario internacional. Este artículo tiene como objetivo analizar la cooperación internacional para el control del tráfico de armas, municiones y explosivos como un mecanismo para la generación de seguridad ontológica en Colombia, para lo cual se va a hacer uso de un enfoque teórico constructivista.

Palabras clave: seguridad ontológica, tráfico de armas, municiones y explosivos, esquemas de rutinización, mecanismos de cooperación internacional, identidad.

\title{
INTERNATIONAL COOPERATION FOR CONTROL OF TRAFFIC OF FIREARMS, AMMUNITION AND EXPLOSIVES: A MECHANISM FOR THE GENERATION OF ONTOLOGICAL SECURITY IN COLOMBIA
}

\begin{abstract}
Although the results of the Colombian participation in international cooperation schemes aimed at the control of traffic of firearms, ammunition and explosives, are generally analyzed regarding physical aspects such as reduction of homicides and impounding of illegal weapons, it is necessary to identify other areas equally important for implementing security in the country; among these are the aspects of identity and recognition of Colombia's position in the international scene. This article aims to analyze international cooperation for control of traffic of firearms, ammunition and explosives as a mechanism for the generation of ontological security in Colombia, for which we are going to use a constructivist theoretical approach.
\end{abstract}

Key words: ontological security, traffic of firearms, ammunition and explosives, routinization schemes, international cooperative mechanisms, identity.

\section{COOPERAÇÃO INTERNACIONAL PARA O CONTROLE DO TRÁFICO DE ARMAS, MUNIÇÕES E EXPLOSIVOS: UM MECANISMO PARA A GERAÇÃO DA SEGURANÇA ONTOLÓGICA NA COLÔMBIA.}

\section{RESUMO}

Embora os resultados da participação da Colômbia nos mecanismos de cooperação internacional que visam controlar o tráfico de armas, munições e explosivos, geralmente sejam analisados em relação aos efeitos físicos em termos de redução de homicídios e da apreensão de armas ilegais, é necessário identificar outras áreas igualmente importantes para a construção da segurança no país, as quais incluem o aspecto da identidade e reconhecimento da posição da Colômbia no cenário internacional. O objetivo deste artigo é analisar a cooperação internacional para controlar 
o tráfico de armas, munições e explosivos como um mecanismo para a geração da segurança ontológica na Colômbia, o qual utilizará uma abordagem teórica construtivista.

Palavras-chave: segurança ontológica, tráfico de armas, munições e explosivos, sistemas de rotinização, mecanismos de cooperação internacional, identidade.

\section{INTRODUCCIÓN}

Si bien es cierto que el tráfico de armas, municiones y explosivos ha sido identificado a lo largo del tiempo como un fenómeno preocupante para Colombia y la comunidad internacional, según lo expresado por organizaciones no gubernamentales tales como Small Armas Survey (2006), en el contexto que actualmente vive el país ${ }^{1}$, se requieren unas condiciones claras y eficientes que permitan el control efectivo de esta problemática, teniendo en cuenta la posibilidad de una salida negociada al conflicto armado y la necesidad de control del material bélico flotante que se genera a partir del mismo.

Dicha necesidad surge de la búsqueda de herramientas para la prevención de reincidencia en prácticas delincuenciales y la repetición de ciclos de violencia (Observatorio de procesos de DDR, 2010), lo cual, junto con los incentivos económicos asociados a las actividades ilícitas y la ausencia de unas reglas de juego claras, evidenciado en la falta de una política de Estado sobre control de armas, se establecen como las principales causas de posibles fracasos en escenarios de pos-conflicto (Valencia, 2007).

En este sentido, es importante señalar que Colombia es partícipe de diferentes mecanismos de cooperación internacional para la lucha contra el tráfico de armas, municiones y explosivos tanto en el ámbito regional como internacional, lo cual ha llevado al desarrollo de herramientas jurídicas y operativas que han generado una mayor capacidad de control de este fenómeno, teniendo en cuenta la condición transnacional con la que se presenta y las diversas esferas que afecta.

Dentro de dichos mecanismos se destacan: el "Programa de acción para prevenir, combatir y eliminar el tráfico ilícito de armas pequeñas y ligeras en todos sus aspectos", vigente desde el año 2001 y suscrito en el marco de la Organización de Naciones Unidas; la "Convención

1. Colombia tiene un grave problema de control de armas, generado por la gran capacidad de tráfico de armas, municiones y explosivos de los grupos insurgentes y de las bandas criminales. En el lapso de enero a septiembre de 2009, las Fuerzas Militares incautaron 4.400 armas pequeñas y ligeras, 1'206.708 cartuchos de diferentes calibres y 7.005 granadas de diferentes tipos; así mismo, en el año 2007, la Policía Nacional de Colombia incautó 56.634 armas pequeñas y ligeras y 722.143 municiones de diferentes calibres, según fuentes del Ministerio de Defensa. 
Interamericana contra la fabricación y el tráfico ilícitos de armas de fuego, municiones, explosivos y otros materiales relacionados" (CIFTA), vigente desde el año 1997 y contemplada en el esquema institucional de la OEA; la "Decisión 552 del Consejo Andino por el que se aprueba el Plan Andino para la prevención, combate y erradicación del tráfico ilícito de armas pequeñas y ligeras en todos sus aspectos", vigente desde el año 2003 y la "Decisión 7/98 del Consejo del Mercado Común, por la que se aprueba el Mecanismo Conjunto de registro de compradores y vendedores de armas de fuego, municiones, explosivos y otros materiales relacionados, vigente desde 1998 (Ardila, 2012: 71).

Los resultados de este tipo de mecanismos, generalmente son analizados en relación con los efectos físicos en términos de la reducción de homicidios y el incautamiento de armas ilegales por parte de las autoridades competentes. Sin embargo, es importante que se identifiquen otras esferas igualmente importantes para la construcción de seguridad en el país, dentro de los que se incluye el aspecto identitario considerado desde una perspectiva subjetiva e intersubjetiva, lo cual va a llevar a la consolidación y el reconocimiento de una posición de Colombia en el escenario internacional. En este proceso, la capacidad de predicción y el saber cómo actuar y cómo identificarse a sí mismo frente a determinados contextos, se establece como una condición prioritaria a la cual no siempre se le da la relevancia que debería tener tanto en el ámbito académico como en el diseño de políticas públicas.

Este artículo tiene como objetivo analizar la cooperación internacional para el control del tráfico de armas, municiones y explosivos como un mecanismo para la generación de seguridad ontológica en Colombia. Para llevar a cabo este objetivo, inicialmente se buscará entender la dinámica de cooperación internacional desde la perspectiva teórica constructivista, para después aludir a las diferencias entre el concepto de seguridad ontológica y seguridad física.

A partir de esto, se emplearán las medidas y los resultados obtenidos a través del programa de acción de las Naciones Unidas y de la decisión 552 del Plan Andino para identificar cómo se generan las condiciones que permiten el establecimiento de un escenario de seguridad ontológica en Colombia y qué beneficios representa para la construcción de una identidad colectiva positiva en el sistema internacional.

\section{LOS MECANISMOS DE COOPERACIÓN INTERNACIONAL PARA EL CONTROL DEL TRÁFI- CO DE ARMAS DESDE LA PERSPECTIVA TEÓRICA CONSTRUCTIVISTA}

La cooperación internacional como un fenómeno relevante en las relaciones internacionales ha sido analizada desde diferentes perspectivas teóricas, con el propósito de entender cuál es la motivación y el interés de los Estados en la participación de este tipo de mecanismos a partir de postulados específicos que plantean herramientas y visiones concretas de la realidad. 
Sin embargo, en términos generales, se puede plantear que la cooperación internacional es entendida como "la interacción creativa entre los Estados, la promoción del diálogo y el acercamiento para resolver problemas comunes a partir del entendimiento y no del enfrentamiento" (Instituto Mexicano de Cooperación internacional, 11).

Esta definición del concepto de cooperación internacional, aunque plantea el objetivo de dicha dinámica, no proporciona los elementos para entender cómo se genera y qué factores inciden en la misma. De acuerdo a esto, es importante señalar que desde el realismo político, la cooperación internacional obedece a una estrategia de política exterior a través de la cual se busca concretar el interés nacional de los Estados involucrados, teniendo en cuenta que estos se caracterizan por una condición racional y egoísta, lo cual incide en su comportamiento dentro del escenario internacional (Buchelli, 2008).

A diferencia de esta perspectiva, el enfoque teórico constructivista considera el proceso de construcción de identidad bajo parámetros de interacción, como un factor prioritario tanto en la consolidación de intereses como en la decisión de participación en los diferentes mecanismos de cooperación internacional que se generan alrededor de diversos temas. De ese modo, dentro este marco de análisis, la identidad es entendida como "a subjective or unit-lev quality, rooted in an actor's self-understandings" (Wendt, 1999: 224), consideración que sólo va adquirir sentido en relación con el significado colectivo que los otros actores reconozcan en escenarios de interacción.

Es pertinente señalar que existen cuatro tipos de identidades que van a permitir establecer la relación entre la identificación subjetiva de Colombia como un Estado social de derecho con las condiciones coyunturales y estructurales tanto en el ámbito nacional como internacional: "la identidad personal, que hace referencia a las propiedades esenciales que definen a los Estados como tal; la identidad de tipo, concerniente a la concepción cognitiva de cómo se reconoce el Estado a sí mismo de acuerdo a determinadas características; la identidad de rol, que hace alusión a la internalización de la identidad de tipo en una estructura social y depende de los patrones de interacción e intersubjetividad; y finalmente, la identidad colectiva que comprende la coherencia entre la identidad de tipo y la identidad de rol" (Peña, 2012).

En este sentido, es el proceso de construcción de identidad lo que se establece como un factor determinante en la consolidación de intereses ${ }^{2}$ y en la toma de decisiones y comportamientos estatales. Es así como la percepción común de la realidad y la identificación de un fenómeno como una amenaza compartida, lleva a los Estados a la búsqueda de espacios de cooperación internacional, los que a su vez permiten el diseño y ejecución de mecanismos efectivos que consideran la condición transnacional de las amenazas.

2. Entendidos como objetivos deseables (Wendt, 1999: 122). 
Como evidencia de esto, el programa de acción de las Naciones Unidas se establece a partir de la preocupación compartida por "la fabricación, transferencia y circulación ilícitas de armas pequeñas y ligeras y por su acumulación excesiva y su proliferación incontrolada en muchas regiones del mundo, lo cual tiene consecuencias humanitarias y socioeconómicas de muy diversa indole y supone una grave amenaza para la paz, la reconciliación, la seguridad, la estabilidad y el desarrollo sostenible en los planos individual, local, nacional, regional e internacional" (Naciones Unidas, 2001: 6).

Dicha preocupación generada como producto de una percepción común de la realidad y unida con el reconocimiento de la paz y la seguridad como factores indispensables para el desarrollo sostenible en los planos individual, nacional, regional e internacional, constituyen un contexto favorable para el establecimiento de los mecanismos de cooperación internacional, entendidos como canales de comunicación institucionalizados (Gilson, 2002: 16). Las principales diferencias entre la cooperación internacional analizada desde una perspectiva constructivista y un enfoque racionalista se presentan en la siguiente tabla:

TABLA 1. Diferencias entre la cooperación internacional entendida desde la perspectiva constructivista y el enfoque racionalista

\begin{tabular}{|l|l|l|}
\hline & \multicolumn{1}{|c|}{$\begin{array}{c}\text { ACCIÓN COMUNICATIVA } \\
\text { (constructivismo) }\end{array}$} & $\begin{array}{c}\text { ACCIÓN ESTRATÉGICA } \\
\text { (racionalismo) }\end{array}$ \\
\hline Orientación, objetivo & $\begin{array}{l}\text { Entendimiento mutuo, creación } \\
\text { de interpretaciones comunes de } \\
\text { la realidad. }\end{array}$ & $\begin{array}{l}\text { Éxito, realización de la optimi- } \\
\text { zación de las preferencias indi- } \\
\text { viduales. }\end{array}$ \\
\hline $\begin{array}{l}\text { Modo de coordinación } \\
\text { social (comportamiento) }\end{array}$ & Argumentos persuasivos & Incentivos externos \\
\hline Carácter & Contingente, dinámico & Estático \\
\hline
\end{tabular}

Fuente: Schünemann (2006: 193)

Sin embargo, además de la importancia de las identidades e intereses, la condición anárquica del sistema internacional ${ }^{3}$ (Waltz, 1988) representa un factor relevante para la construcción de seguridad por parte de los Estados. De acuerdo a esto, es importante tener en cuenta que dicho

3. Determinado por la ausencia de un poder ordenador y el protagonismo que han ido adquiriendo los actores no estatales en dicho contexto (Peña, 2012: 7). 
proceso implica no solo el empleo de herramientas dirigidas a la disminución de amenazas físicas, sino también a la búsqueda de mecanismos que generen eficiencia en el cumplimiento de ese objetivo, a partir del establecimiento de patrones de predictibilidad que reduzcan la ansiedad y la incertidumbre que por lo general caracterizan el escenario internacional.

\section{LA SEGURIDAD ONTOLÓGICA Y LA SEGURIDAD TRADICIONAL EN PERSPECTIVA COMPARADA}

La seguridad siempre ha representado un tema de gran importancia en la jerarquía de intereses de las entidades estatales en relación con temas tan diversos como la economía, los conflictos inter-estatales, los conflictos internos, la protección de la soberanía, el terrorismo, las armas no convencionales, entre otros. Es así como en términos generales este concepto hace referencia a la disminución de amenazas hacia valores prioritarios, especialmente aquellos que amenazan la supervivencia de un objeto referente en un futuro cercano (Williams, 2008: 5).

Bajo este marco de análisis, tanto la importancia de los objetos referentes como la percepción de amenaza hacia estos, va a estar determinada de acuerdo con los significados colectivos, consolidados a través de la identidad y de las dinámicas de interacción con otros actores. Es así como una situación que puede representar un posible riesgo para cierto Estado, no necesariamente tiene el mismo significado para otro, lo cual ha sido un aspecto trascendente y constante en las razones que han motivado a la participación de diferentes países en espacios de cooperación internacional como lo son el Programa de Acción de las Naciones Unidas y el Plan Andino, dirigidos al control del tráfico de armas, municiones y explosivos.

El término de seguridad ontológica fue introducido por Jeffrey Huysmans en 1998 y es definido como "la condición que se obtiene cuando un actor tiene expectativas confiables, incluso si son probabilidades acerca de los medios y los fines de las relaciones que gobiernan su vida social" (Mitzen, 2006: 345). En este sentido, la seguridad ontológica está asociada con "la capacidad de predicción y el saber cómo actuar y cómo identificarse a sí mismo frente a determinados contextos" (Peña, 2012: 8).

A partir de esto, surge la principal diferencia entre la seguridad vista desde un sentido tradicional y la seguridad considerada desde un enfoque ontológico. Con respecto a la primera, esta tiene como objetivo fundamental la garantía de supervivencia, mientras que desde la esfera ontológica, es la posibilidad de predicción y el reconocimiento de los otros en relación con la identidad propia, lo que va representar un aspecto prioritario. Es pertinente señalar que estas dos perspectivas no son componentes excluyentes dentro del esquema de intereses de las entidades estatales, sino que tienen una relación complementaria. 
Por otra parte, en alusión a la percepción que se produce al enfrentar una amenaza o una fuente de inseguridad, la sensación que se genera desde el punto de vista de la seguridad tradicional es el miedo a la posibilidad de daño físico, cuyo resultado sería un cambio estructural en la distribución de poder, mientras que desde la perspectiva de la seguridad ontológica, es la ansiedad y la incertidumbre lo que podría llegar a consolidar situaciones críticas, entendidas como "circunstancias de disyunción radical que afectan un número importante de personas y generan situaciones que amenazan o destruyen las certezas de las rutinas institucionalizadas" (Steele, 2008: 51).

De acuerdo a esto, "la seguridad ontológica se genera a partir de una estabilidad cognitiva con respecto a determinado contexto, lo cual va a permitir una reducción de costos de transacción" (Peña, 2012: 10), que en este caso aluden al procesamiento de amenazas y diseño de estrategias en el continente americano con respecto al fenómeno del tráfico de armas, municiones y explosivos.

Es así como es importante analizar las condiciones y herramientas proporcionadas por los mecanismos de cooperación internacional dirigidos hacia este objetivo, con el fin de identificar el marco contextual a partir del cual se generan escenarios de seguridad no solo desde un sentido físico sino también ontológico. Para llevar a cabo este propósito, en el siguiente apartado se hará referencia al "Programa de acción para prevenir, combatir y eliminar el tráfico ilícito de armas pequeñas y ligeras en todos sus aspectos", vigente desde el año 2001 y suscrito en el marco de la Organización de Naciones Unidas y a la "Decisión 552 del Consejo Andino por el que se aprueba el Plan Andino para la prevención, combate y erradicación del tráfico ilícito de armas pequeñas y ligeras en todos sus aspectos".

\section{LOS MECANISMOS DE COOPERACIÓN INTERNACIONAL CONTRA EL TRÁFICO DE ARMAS COMO MEDIO PARA LA GENERACIÓN DE SEGURIDAD ONTOLÓGICA}

El contexto de seguridad de Colombia durante los años noventa del siglo pasado, estaba caracterizado por un incremento de los miembros correspondientes a los grupos armados al margen de la ley y un aumento de las prácticas asociadas a la actividades delictivas del narcotráfico (Ardila, 2012: 75). Dichos fenómenos incidieron de forma directa en la tasa de homicidios calculada en un $89.5 \%$ para inicios de esta década, cifra que descendió a un $56.4 \%$ para el año 1998 (Observatorio del programa presidencial de Derechos Humanos y DIH, 2009: 11).

La confluencia de estos factores configuró un escenario negativo para Colombia situándolo como uno de los países más violentos de América Latina. Sin embargo, es importante destacar que para este periodo de tiempo, el fenómeno del tráfico de armas ya era considerado como un tema prioritario y percibido como una amenaza común en el hemisferio occidental. Como evidencia de ello, en el año 1997 se diseñó y adoptó la Convención Interamericana contra la Fabricación y el 
Tráfico llícito de Armas de Fuego, Municiones, Explosivos y Materiales Relacionados (CIFTA), bajo la estructura institucional de la Organización de Estados Americanos (Godnick, 2006: 9).

Posteriormente, en el año 2001, a partir de la identificación del tráfico de armas, municiones y explosivos como una amenaza para la estabilidad y el desarrollo sostenible en los planos individual, local, nacional, regional e internacional (Naciones Unidas, 2001: 6), se establece el "Programa de acción para prevenir, combatir y eliminar el tráfico ilícito de armas pequeñas y ligeras en todos sus aspectos", con el objetivo de desarrollar herramientas jurídicas y operativas que representaran una alternativa efectiva frente a un problema de carácter transnacional.

Así las cosas, desde el marco de análisis de la seguridad ontológica, la definición concreta de lo que se entiende por armas pequeñas y ligeras, así como la disposición de estrategias de acción que debían ser asumidas por los países participantes, se consideran como una construcción de espacios por medio de los cuales se generan escenarios de estabilidad cognitiva, en los que los Estados además de reducir los costos de transacción derivados del procesamiento de amenazas, evitan el diseño general de las disposiciones que deben ser implementadas para afrontar fenómenos como el tráfico de armas.

Con respecto a la definición, dentro de la guía práctica de la legislación sobre armas pequeñas y ligeras se señala que este término hace referencia a

"cualquier arma letal que pueda llevar una persona y que expulse o lance, esté diseñada para expulsar o lanzar o pueda convertirse fácilmente para expulsar o lanzar una bala o proyectil por la acción de un explosivo, excluyendo armas pequeñas y armas ligeras de anticuario o sus réplicas. Las armas pequeñas y las armas ligeras de anticuario y sus réplicas se definirán de acuerdo con las leyes nacionales. En ningún caso las armas pequeñas y ligeras de anticuario incluirán las fabricadas después de 1899" (Programa de desarrollo de las Naciones Unidas, 2008: 6).

En lo que alude a las estrategias de acción propuestas por este mecanismo de cooperación internacional es importante destacar: el fortalecimiento de la estructura jurídica interna; la recolección y destrucción de armas de fuego; el establecimiento de un programa de desarme; el avance en el registro detallado de armas circulantes en cada país; la obligatoriedad de la huella balística para los importadores; la ejecución de programas de sensibilización; el intercambio constante de información asociada al tráfico de armas y la participación de la sociedad civil (Ardila, 2012: 29).

En este sentido, tanto la identificación del tráfico de armas como un fenómeno preocupante como la disposición de herramientas para afrontarlo eficazmente, demuestra una voluntad política por parte de Colombia que se traduce en un tipo de "acción honorable", determinada por la búsqueda de reputación o credibilidad en beneficio de la consecución de los intereses 
nacionales (Steele, 2008: 32). De acuerdo a esto, la participación en mecanismos de cooperación internacional dirigidos al control del tráfico de armas, municiones y explosivos no solo va a significar la posibilidad de una reducción de homicidios cometidos desde la esfera de la ilegalidad, lo cual representa un beneficio en términos físicos, sino que además implica la proyección de Colombia como un país líder en la proposición de alternativas para la lucha y el control de este problema, condición que efectivamente ha sido reconocida en los diferentes espacios de interacción internacional (Unodc, 2005).

Otra fuente importante que debe ser considerada en la generación de seguridad ontológica, es el establecimiento de esquemas de rutinización, ya que por medio de estos se consolidan prácticas habituales que facilitan la capacidad de reacción y el reconocimiento de un Estado frente a determinado contexto. De esta forma, una de las disposiciones señaladas por el programa de acción de las Naciones Unidas hace referencia a la entrega periódica de un informe de cumplimiento, en el que los diferentes países miembros dan cuenta de los avances alcanzados en diversas áreas temáticas y en aspectos concretos que resultan indispensables para el logro del objetivo propuesto en el marco de dicho mecanismo.

En el caso de Colombia, es pertinente señalar la creación del Comité de Coordinación Nacional para la Prevención, Combate y Erradicación del Tráfico llícito de Armas Pequeñas y Ligeras en todos sus Aspectos como el Organismo Nacional de Coordinación, mediante el Decreto expedido el 19 de diciembre de 2006. Dicho comité tiene como funciones principales:

"el diseño e implementación del Plan Nacional de acción; la orientación de las políticas, la investigación y el monitoreo de la proliferación, control y tráfico de armas; la búsqueda de unificación de las bases de datos disponibles acerca de las armas pequeñas y ligeras que existen en el país; el suministro de información oficial requerido por otros comités e instituciones vinculadas con el tema, entre otras tareas" (Ardila, 2012: 49).

Después de la creación del Comité identificado como el Organismo Nacional de Coordinación, Colombia ha publicado dos informes de aplicación correspondientes al programa de acción de las Naciones Unidas, uno en el año 2008 y otro en el año 2010. Dentro de los principales indicadores de cumplimiento frente a los cuales Colombia ha reportado avances están: las leyes y los procedimientos para el control sobre armas pequeñas y ligeras tanto en el ámbito internacional como nacional; la aplicación coercitiva de la ley y la tipificación de delitos; las normas y procedimientos nacionales en relación con la gestión y seguridad de almacenamiento; el esquema de marcación, mantenimiento, registro y localización y el manejo de armas, en cuanto al número de material bélico incautado, entregado y erradicado.

A partir de esto, los principales resultados obtenidos en términos físicos fueron: un destacado sistema de marcación, identificado como uno de los más completos del hemisferio al tener en cuenta la marcación individual de las municiones y explosivos por lotes; el establecimiento de 
un amplio marco jurídico por medio del cual se regulan todas aquellas actividades asociadas al manejo del material bélico y la disposición de los elementos operativos materializados en diferentes sistemas de información tales como el manual IM OC DAC MN 001 (para armas y municiones), el Sistema de Información de Armas, municiones y explosivos (SIAEM) y el Centro de Información Nacional de Armas (CINAR) (Ministerio de Relaciones Exteriores, 2008-2010).

En términos de la imagen de Colombia, esta mejoró sustancialmente pasando de ser reconocida como un país con problemas de seguridad, que representaban una amenaza a la agenda internacional a inicios de la década del 2000 (El Tiempo, 2000) a un país con importantes posibilidades de desarrollo económico y social en la región. Es pertinente señalar que los beneficios obtenidos en esta área no pueden ser atribuidos en su totalidad a la participación de Colombia en los mecanismos de cooperación internacional, sino que es necesario identificar la incidencia que han tenido factores como la implementación de la Política de Seguridad Democrática y el cambio en la misma dinámica del conflicto en la disminución de la tasa de homicidios cometida con armas de fuego.

Con respecto al Plan Andino para prevenir, combatir y erradicar el comercio ilícito de APL, adoptado el 25 de junio de 2003, es importante destacar que este se crea con el propósito de complementar el Programa de Acción de las Naciones Unidas, teniendo en cuenta las particularidades propias de la región Andina y la definición del concepto de seguridad, entendido como:

"La situación en la que el Estado y la sociedad se encuentran protegidos frente a amenazas o riesgos susceptibles de afectar el desarrollo integral y el bienestar de sus ciudadanos, así como el libre ejercicio de sus derechos y libertades en un contexto de plena vigencia democrática. En ese sentido, la seguridad es un concepto de carácter multidimensional y comprehensivo que abarca asuntos de índole política, económica, social y cultural, y se ve reflejada en las políticas en ámbitos tan diversos como los del fortalecimiento de la institucionalidad democrática y el estado de derecho, la defensa, la salud, el ambiente, la economía, el desarrollo económico y la prevención de desastres naturales, entre otros" (Comunidad Andina, 2003).

De acuerdo a esto, es evidente la relación que existe entre el concepto de seguridad considerado desde la perspectiva andina y la identidad de tipo de Colombia, que hace referencia a la concepción subjetiva del Estado a partir de ciertas características. Dicha concepción se ve reflejada en el artículo primero del Título I de la Constitución Política, en dónde se plantea que:

"Colombia es un Estado social de derecho, organizado en forma de República unitaria, descentralizada, con autonomía de sus entidades territoriales, democrática, participativa y pluralista, fundada en el respeto de la dignidad humana, en el trabajo y la solidaridad de las personas que la integran y en la prevalencia del interés general" (Constitución Política de Colombia, 1991). 
En estos dos planteamientos (la definición del concepto de seguridad y el artículo primero del Título I), el contexto democrático, el Estado de derecho y el respeto a la condición de pluralidad se establecen como elementos prioritarios, a partir de los cuales se genera un marco de interpretación común de la realidad, que sugiere una perspectiva compartida con respecto a un área temática y en particular dentro de un límite geográfico específico. Es así como la participación de Colombia en el Plan Andino para prevenir, combatir y erradicar el comercio ilícito de APL, refuerza el tipo de "acción honorable", por medio de la cual el país demuestra estar interesado en la defensa de unos valores que se identifican como prioritarios para la estabilidad y el desarrollo político, económico y social.

\section{CONSOLIDACIÓN DE UNA IDENTIDAD COLECTIVA POSITIVA PARA COLOMBIA}

Es importante tener en cuenta que si bien los esquemas de rutinización se identifican como un mecanismo generador de seguridad ontológica, esta condición no implica la garantía de seguridad física. En este sentido, con respecto a los resultados obtenidos en términos materiales, se destaca un aumento de las armas incautadas por parte de la policía, pasando de 15.637 en el año 2000 a 25.453 y 20.967 para el año 2004 y 2005 respectivamente (ONU, 2005: 59). Por otra parte, aunque no es posible establecer una relación directamente proporcional entre la concertación de espacios de cooperación internacional dirigidos al control del tráfico de armas y la reducción del índice de homicidios, es evidente que en la medida en que se limite la disposición de material bélico, se va a promover a su vez una disminución de muertes causadas por este tipo de armas. Así las cosas, el índice de homicidios que estaba calculado en un 89.5\% para inicios de la década del noventa, descendió a un $17.4 \%$ para lo que va corrido del año 2012 (Oficina de Prensa, Alcaldía Mayor de Bogotá, 2012).

En alusión a los resultados obtenidos en términos ontológicos, la consolidación de mecanismos de rutinización generados en el marco de los espacios de cooperación internacional, tales como el establecimiento de un comité de coordinación nacional; el diseño e implementación de un plan nacional; la tipificación penal para fabricación ilícita, tráfico ilícito, tenencia ilícita, porte ilícito y almacenamiento ilícito; la existencia de un sistema de licencias de importación/exportación; la disposición de legislación sobre incautación/confiscación; la destrucción de inventarios excedentes de APL del Estado, así como de las armas incautadas o recolectadas de particulares y la existencia de un órgano de registro (de licencias de posesión, de importadores, comercializadores y de empresas de seguridad privada) (Oficina de Asuntos de Desarme de las Naciones Unidas, 2010), se traducen en las condiciones y herramientas necesarias para la construcción de un sistema de confianza básico, entendido como "un capullo cognitivo que funciona como soporte para el conocimiento de los posibles eventos en los cuales se pueda ver amenazada la integridad física o psicológica de un agente" (Mitzen, 2006: 346).

LA COOPERACIÓN INTERNACIONAL PARA EL CONTROL DEL TRÁFICO DE ARMAS, MUNICIONES Y EXPLOSIVOS: UN MECANISMO PARA LA GENERACIÓN DE SEGURIDAD ONTOLÓGICA EN COLOMBIA 
La disposición de dicho sistema de confianza facilita el establecimiento de condiciones de predictibilidad y la disminución de incertidumbre frente al fenómeno del tráfico de armas. En este sentido, a medida que este sistema se fortalece a través de un proceso de aprendizaje colectivo, se promueve una reducción de costos de transacción ${ }^{4}$ para Colombia, en relación con el procesamiento de amenazas y la aplicación de medidas específicas de prevención y control de esta problemática. Medidas que han sido concertadas con antelación en los mecanismos de cooperación internacional como lo son el Programa de Acción de las Naciones Unidas y la Decisión 552 que dio origen al Plan Andino.

A partir de esto, es evidente que tanto los resultados obtenidos en términos físicos como ontológicos han tenido incidencia en la imagen proyectada por Colombia en el sistema internacional y en el proceso de consolidación de una identidad colectiva congruente con el reconocimiento subjetivo de Colombia como un Estado Social de Derecho (Constitución Política de Colombia, 1991). Esta condición se ve materializada en el cambio de las consideraciones que actores como la Organización de Naciones Unidas manifestaban tener con respecto a la situación de seguridad del país en el año 1997, momento en el cual planteaban que:

“Colombia continúa sufriendo de una de las tasas de homicidios más altas del mundo. Dentro del contexto de violencia debe hacerse referencia a la criminalidad generada por las organizaciones de narcotraficantes, que incluso han llegado a ofensivas terroristas, mediante la colocación de explosivos en sitios públicos. Existen indicios sobre el apoyo que estas organizaciones prestan a los grupos paramilitares, que en ocasiones actúan como ejércitos privados de los grandes jefes de los carteles de la droga" (Naciones Unidas, 1997: 4).

La mejoría que tuvo la situación de seguridad en Colombia y la preocupación demostrada por el país en el diseño y ejecución de mecanismos dirigidos a la garantía de los principios constitucionales, se vio reflejada en un cambio de percepción por parte de la comunidad internacional, lo cual se evidencia en el informe presentado por la Alta Comisionada de las Naciones Unidas, quién en el año 2011 señaló:

"En 2011 se abrieron y ampliaron espacios políticos para el trabajo de promoción y protección de los derechos humanos. La Alta Comisionada valora los esfuerzos del Gobierno para fortalecer el estado de derecho. El Gobierno emprendió importantes iniciativas legislativas y de política pública, condenó una serie de violaciones de derechos humanos y tomó acción contra la corrupción y el despojo de tierras. Sin embargo, hasta ahora, estos esfuerzos no han logrado el impacto deseado a nivel local" (Naciones Unidas, 2012).

4. Hace referencia a los costos en los que se incurre para obtener información, seguridad, legalidad, confianza y garantías (Kalmanovitz, 2003: 8). 
Ahora bien, aunque la apropiación de condiciones de seguridad ontológica han favorecido otros procesos asociados al desarrollo del país, como lo son el aumento del flujo de la inversión extranjera que pasó de 4.000 millones de dólares en al año 1999 (El Tiempo, 1999) a 10.564 en el año 2008 (Portafolio, 2009), es importante destacar que Colombia, y en general América Latina, sigue siendo una región prioritaria para entender el fenómeno del tráfico de armas, teniendo en cuenta que es aquí donde suceden el $40 \%$ del total de muertes producidas por armas de fuego (Godnick, 2006: 2). En este sentido, es necesario seguir avanzando en la búsqueda de mecanismos efectivos que aprovechen los procesos cognitivos derivados de las experiencias en esta área pero a que a su vez se adapten a las condiciones cambiantes del contexto nacional, regional y global.

\section{CONCLUSIONES}

La participación de Colombia en los mecanismos de cooperación internacional para el control del tráfico de armas, municiones y explosivos, debe ser analizada desde una perspectiva integral que considere los efectos a mediano y largo plazo en relación con el interés nacional de Colombia y con un reconocimiento del país como un Estado Social de Derecho. En este sentido, tal y como se pretendía demostrar en este artículo, es importante destacar que la seguridad física y la seguridad ontológica son escenarios cuyas condiciones generan efectos complementarios, razón por la cual, a medida que se fortalezca el desarrollo de herramientas operativas y jurídicas, alimentadas a partir de procesos de rutinización, se va a promover a su vez un contexto de seguridad ontológica, en el que se aumentan los patrones de predictibilidad y se proyecta una imagen positiva de Colombia en el escenario internacional.

Así las cosas, el establecimiento de mecanismos de cooperación internacional para el control del tráfico de armas tales como el Programa de Acción de las Naciones Unidas y el Plan Andino, permiten la consolidación de un sistema de confianza que proporciona una estabilidad cognitiva con respecto a posibles eventos, cuyo desarrollo puede representar una fuente de amenaza física o psicológica para un agente. Es así como dicho sistema se fortalece a partir de la implementación de un esquema de rutinización, que en este caso alude a las medidas dispuestas en el marco institucional de los mecanismos de cooperación internacional, por medio de los cuales se promueve una reducción de los costos de transacción en relación con los escenarios que se perciben como una amenaza en el ámbito nacional y regional, así como en relación con las herramientas que en términos generales deben ser empleadas para afrontar las mismas.

De acuerdo a esto, la participación de Colombia en espacios de cooperación internacional dirigidos al control del tráfico de armas, no solo implica un beneficio físico evidenciado en la reducción de la tasa de homicidios cometidos con armas de fuego y de la incautación y 
eliminación de este tipo de material bélico, sino que también genera un efecto positivo para el reconocimiento de Colombia como un Estado Social de Derecho, preocupado por la garantía de las disposiciones constitucionales y por la búsqueda del cumplimiento de unos objetivos compartidos en el continente americano, lo cual va a significar un aumento de la confianza en el país y un posible desarrollo económico y social derivado de éste.

\section{REFERENCIAS}

- Ardila, C. (2012). Identificación y evaluación de impacto de las medidas aplicadas por parte de Colombia en el marco de los mecanismos de cooperación internacional contra el tráfico de armas, municiones y explosivos. Universidad Militar Nueva Granada, 1-124.

- $\quad$ Buchelli, F. (2008 ). La cooperación internacional descentralizada: ¿un escenario común entre las relaciones internacionales y el desarrollo? Memorias del primer congreso de ciencia política, 1-19.

- $\quad$ Comunidad Andina (2003). Desición 552. Plan Andino para la prevención, combate y erradicación del tráfico ilícito de armas pequeñas y ligeras en todos sus aspectos.

- $\quad$ Constitución política de Colombia (1991). De los principios fundamentales. Recuperado el 5 de agosto de 2011 de: http://www.banrep.gov.co/regimen/resoluciones/cp91.pdf

- Gilson, J. (2002 ). Defining interregionalism. The Asia-Europe meeting. SEAS Electronic Working Papers

- $\quad$ Godnick, W. (2006). El control de las armas pequeñas en América Latina. Recuperado el 12 de febrero de 2012 de http://www.bvsde.paho.org/bvsacd/cd57/godnick.pdf

- Instituto Mexicano de Cooperación Internacional (2006). Apuntes sobre la cooperación internacional internacional para el desarrollo, 11-36. Recuperado el 1 de noviembre de 2012 de http://dgctc.sre.gob.mx/pdf/info2005_capitulo1.pdf

- $\quad$ Kalmanovitz, S. (2003). El neoinstitucionalismo como escuela. Revista de Economía Institucional, 5 (9), 1-22.

- Ministerio de Relaciones Exteriores (2008). INFORME APLICACIÓN EN COLOMBIA DEL PROGRAMA DE ACCIÓN DE LAS NACIONES UNIDAS PARA PREVENIR, COMBATIR Y ELIMINAR EL TRÁFICO ILÍCITO DE ARMAS PEQUEÑAS Y LIGERAS EN TODOS SUS ASPECTOS. Bogotá: Dirección de Asuntos Políticos Multilaterales, Coordinación de Desarme y Seguridad. 
- Ministerio de Relaciones Exteriores (2010). INFORME APLICACIÓN EN COLOMBIA DEL PROGRAMA DE ACCIÓN DE LAS NACIONES UNIDAS PARA PREVENIR, COMBATIR Y ELIMINAR EL TRÁFICO ILÍCITO DE ARMAS PEQUEÑAS Y LIGERAS EN TODOS SUS ASPECTOS. Bogotá: Dirección de Asuntos Políticos Multilaterales, Coordinación de Desarme y Seguridad.

- Mitzen, J. (2006). Ontological security in world politics: State identity and the security dilemma. European Journal of international relations, 341-370.

- $\quad$ Naciones Unidas (1997). Informe de la Alta Comisionada de las Naciones Unidas para los Derechos Humanos sobre la Oficina en Colombia.

- $\quad$ Naciones Unidas (2001). Informe de la Conferencia de las Naciones Unidas sobre el Tráfico Ilícito de Armas Pequeñas y Ligeras en todos sus aspectos. Nueva York. Recuperado el 12 de febrero de 2012 de http://www.un.org/spanish/conferences/smallarms/aconf192_15.pdf

- Naciones Unidas, Oficina contra la droga y el delito (2006). Violencia, Crimen y Tráfico llegal de Armas en Colombia. Bogotá: UN.

- $\quad$ Naciones Unidas (2011). Informe de la Alta Comisionada de las Naciones Unidas para los Derechos Humanos sobre la situación de Derechos Humanos en Colombia durante el año 2011. Recuperado el 10 de mayo de 2012 de http://www.hchr.org.co/documentos einformes/informes/altocomisionado/informes.php3? $\operatorname{cod}=15 \&$ cat $=11$

- $\quad$ Observatorio del Programa Presidencial de Derechos Humanos y DIH (2009). Caracterización del homicidio en Colombia 1995-2006. Bogotá: Scripto Ltda.

- Observatorio de procesos. Recuperado el 12 de febrero de 2012 de http://www.unlirec.org/ OurWork/Documents/NationalStudies/EstudioSubregionalDecision552.pdf

- Peña, M. (2012). La Organización de Cooperación internacional de Shanghái como fuente de seguridad para Rusia en Asia Central. Bogotá: Pontificia Universidad Javeriana, 1-50.

- Progama de Desarrollo de las Naciones Unidas. Legislación sobre armas pequeñas y ligeras. (2008). Guía Práctica. Recuperado el 1o de noviembre de 2012 de http://www.poa-iss.org/kit/Spa_42018_UNDP_int_Web_Rev.pdf

- Schünemann, J. (2008). Algo más que racional: la dimensión social de la cooperación internacional interregional y el interregionalismo en las relaciones internacionales. Documentos de Política Pública: las memorias de los foros 2008, 165-219. 
- $\quad$ Steele, B. (2008). Ontological Security in International Relations.

- $\quad$ UNODC. (2005). Violencia, Crimen y Tráfico Ilegal de Armas en Colombia.

- Valencia, D. (2007). Reconstrucción analítica del proceso de desarme, desmovilización y reinserción con las Autodefensas Unidas de Colombia, 2002-2007. Perfil de Coyuntura Económica, 10, 147-191.

- Waltz, K. (1988). Teoria de la política internacional. Buenos Aires: GEL.

- Wendt, A. (1999). Social Theory of International Politics. NY: Cambridge University Press

- Williams, P. (2008). Security Studies: an introduction. NY.

\section{Recursos electrónicos}

- $\quad$ El Tiempo (1999, 27 de octubre). La inseguridad frena mayores flujos de inversión extranjera. Recuperado el 1 de agosto de 2012 de http://www.eltiempo.com/archivo/documento/MAM-947314

- $\quad$ El Tiempo (2000, 23 de agosto). Esfuerzo Conjunto. Recuperado el 12 de abril de 2012 de http://www.eltiempo.com/archivo/documento/MAM-1285192

- $\quad$ Oficina de Prensa, Alcaldía Mayor de Bogotá (2012, 11 de mayo), Bogotá se consolida como una de las ciudades más seguras del continente. Recuperado el 11 de julio de 2012 de http://www.bogotahumana.gov.co/index.php/noticias/comunicados-de-prensa/975bogota-se-consolida-como-una-de-las-ciudades-mas-seguras-del-continente

\section{Bibliografía consultada no citada}

- $\quad$ Adler, E. (1998). Security Communities. United Kingdom: Cambridge University Press.

- Álvarez, D. (2010). Transferencias de Armas, Violencias y Crimen organizado. En: El control de las transferencias internacionales de Armas. Asociación para políticas públicas. Consejo Argentino para las relaciones internacionales.

- $\quad$ Andrade, G. (2009). Rastreo de armas: perspectivas sobre control, tráfico y uso de armas ilegales en Colombia. Fundación ideas para la paz.

- $\quad$ Buzan, B. (2003). Regions and powers the structure of international security. UK: Cambridge University. 
- Guzzini, S. (2006). Constructivism and international relations Alexander Wendt and his critics. Routledge.

- IEPADES. (2006). Armas pequeñas y desarrollo en sociedades post conflicto. Ciudad de Guatemala: IEPADES.

- Morillas, P. (2007). La seguridad internacional después de la guerra fría ¿Avanzando hacia una doctrina de seguridad humana? CIDOB, 1-6.

- Peña, P. (1994). El tímido control de armas ligeras en la ONU, en la OEA y en grupos regionales latinoamericanos. Revista Colombia Internacional, 28, 25-36.

- $\quad$ Reyes, C. (2011). La amenaza de armas pequeñas y ligeras, municiones y explosivos -APLME. Bogotá: Universidad del Rosario

- $\quad$ Small Arms Survey (2006). La Hidra de Colombia. Las múltiples caras de la violencia armada, 1-31.

- $\quad$ Small Arms Survey (2006). Unfinished business. Oxford: Oxford University Press.

- Small Arms Survey (2010). Transparency Counts: Assessing State Reporting on Small Arms Transfers, 2001-08. Geneva: Institute of International and Develpment Studies.

- Soto, A. (1993). El tráfico illegal de armas: un enfoque económico. Bogotá: CEDE, Universidad de los Andes.

- Weldes, J. (1999). Cultures of insecurity: States, communities, and the production of danger. University of Minnesota Press, 14, 1-33.

- Wendt, A. (2005). La anarquía es lo que los Estados hacen de ella. La construcción social de la política de poder. Revista académica de Relaciones Internacionales, 1-34.

- $\quad$ Organización de Estados Americanos (1997). Convención Interamericana contra la Fabricación y el Tráfico llícitos de Armas de Fuego, Municiones, Explosivos y otros materiales relacionados. Recuperado el 12 de febrero de 2012 de http://www.oas.org/juridico/spanish/ tratados/a-63.html

- $\quad$ Organización de Estados Americanos (2004). PRIMERA CONFERENCIA DE LOS ESTADOS PARTE DE LA CONVENCIÓN INTERAMERICANA CONTRA LA FABRICACIÓN Y EL TRÁFICO ILICITOS DE ARMAS DE FUEGO, MUNICIONES, EXPLOSIVOS Y OTROS MATERIALES RELACIONADOS (CIFTA). Recuperado el 12 de abril de 2012 de http://www.oas.org/es/ centro_noticias/discurso.asp?sCodigo $=04-0043$ 